\title{
RADIO-PISTAGE DE SAUMONS ADULTES (Salmo salar) EN LOIRE
}

\author{
D. BARIL, P. GUENEAU \\ Délégation Régionale n 4 du Conseil Supérieur de la Pêche \\ 112. Faubourg de la Cueille - 86000 POITIERS, France
}

\section{RESUMÉ}

La technique du radio-pistage a été mise en œuvre en 1983 et 1984 sur la Loire, pour étudier le comportement des saumons adultes (Salmo salar) face à deux obstacles : les seuils de Dampierre-enBurly et de Belleville-sur-Loire.

Dix-sept saumons ont été équipés d'un émetteur-radio et quinze franchissements ont pu être observés après des arrêts de migration plus ou moins longs a l'aval des seuils.

La durée de ces arrêts de migration a été étroitement liée à l'évolution du paramètre température, excepté pour deux saumons pour lesquels l'hypothèse d'un stress consécutif au marquage a été retenue.

\section{SUMMARY}

\section{RADIO-TAACKING OF ADULT SALMONS (Salmo salar) IN THE RIVER LOIRE}

Radio-tracking has been used in 1983 and 1984 in the River Loire (France) to study the behaviour of adult salmons (Salmo salar) downstream two weirs, Dampierre-en-Burly and Bellevillesur-Loire.

Seventeen salmons have been tagged with a radio transmitter and fifteen ascents of the weir have been observed after a variable delay in migration below the weir.

The length of this delay in migration has been function of the evolution of temperature except for two salmons for which a stress resulting from tagging was involved.

\section{INTRODUCTION}

Le saumon atlantique (Salmo sa/ar) rencontre quatre obstacles en Loire moyenne lors de sa migration anadrome vers ses frayères sur l'Allier.

Pour mieux connaître son comportement devant ces seuils, la Délégation Régionale du Conseił Supérieur de la Pêche de Poitiers, dans le cadre d'une convention d'étude signée avec Electricité de France (Région d'Equipement TOURS), a mis en œuvre la technique de radio-pistage à hauteur des seuils de Dampierre-en-Burly et de Belleville-sur-Loire.

L'objectif de cette étude, par radio-pistage, était de tenter de cerner les paramètres qui conditionnent la possibilité de franchissement de ces seuils par les saumons adultes et de pouvoir ainsi apprécier leur impact.

\section{MATERIELS ET METHODES}

\subsection{Principe}

La progression, dans un fleuve, d'un saumon équipé d'un émetteur-radio peut être connue grâce à l'utilisation d'une station de réception mobile (récepteur-radio + antenne directionnelle de faible encombrement - Figure 1). 
Bull. Fr. Pêche Piscic. (1986) $302: 86-105-87-$

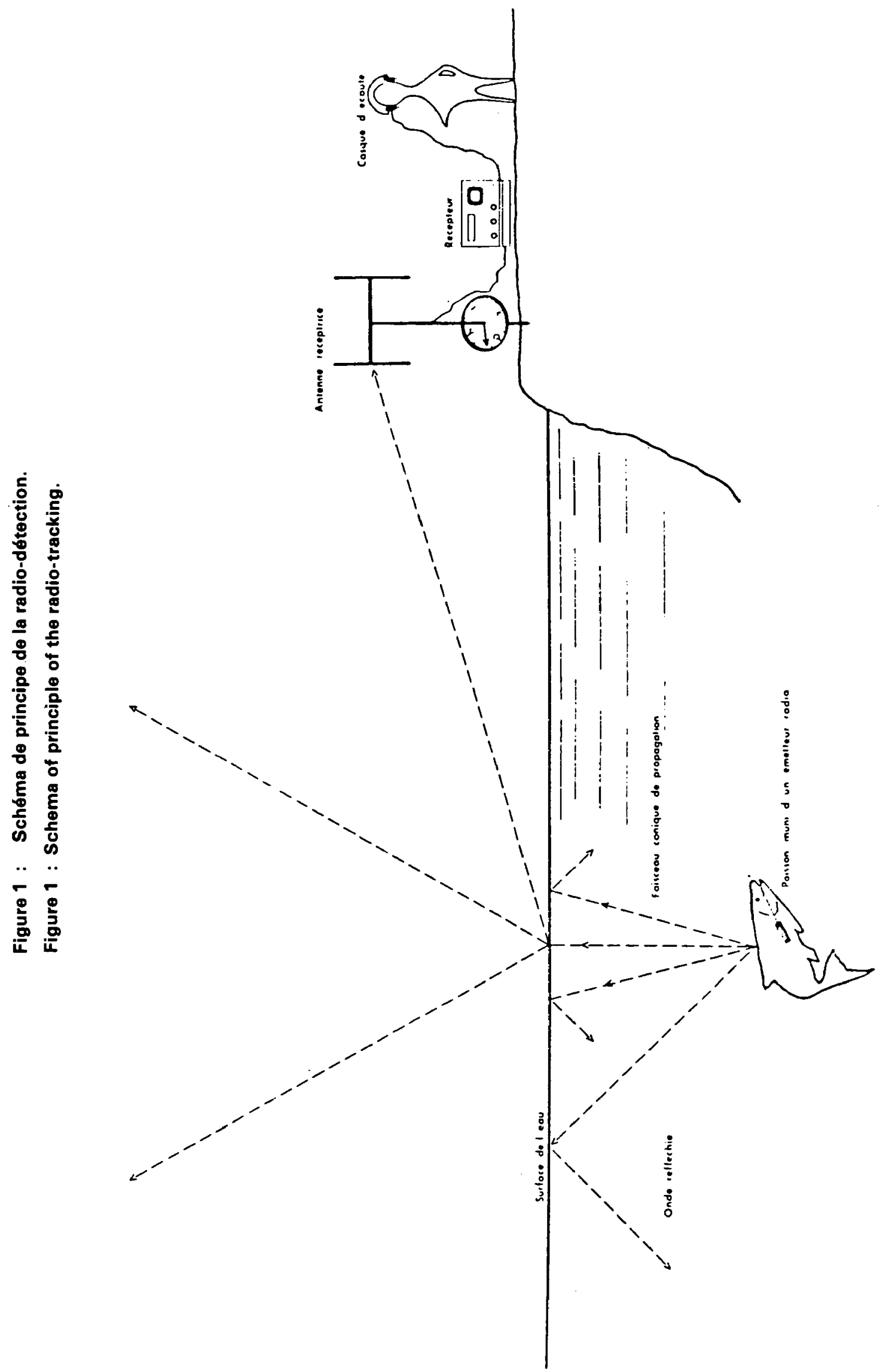


La position de ce même saumon, à l'aval immédiat d'un obstacle, peut être connue grâce au recoupement des directions d'émission du signal radio, recueillies simultanément par deux ou trois stations de réception fixes (poste de réception + grande antenne directionnelle) installées à proximité de l'obstacle (cf. Principe de triangulation, Figure 1 bis).

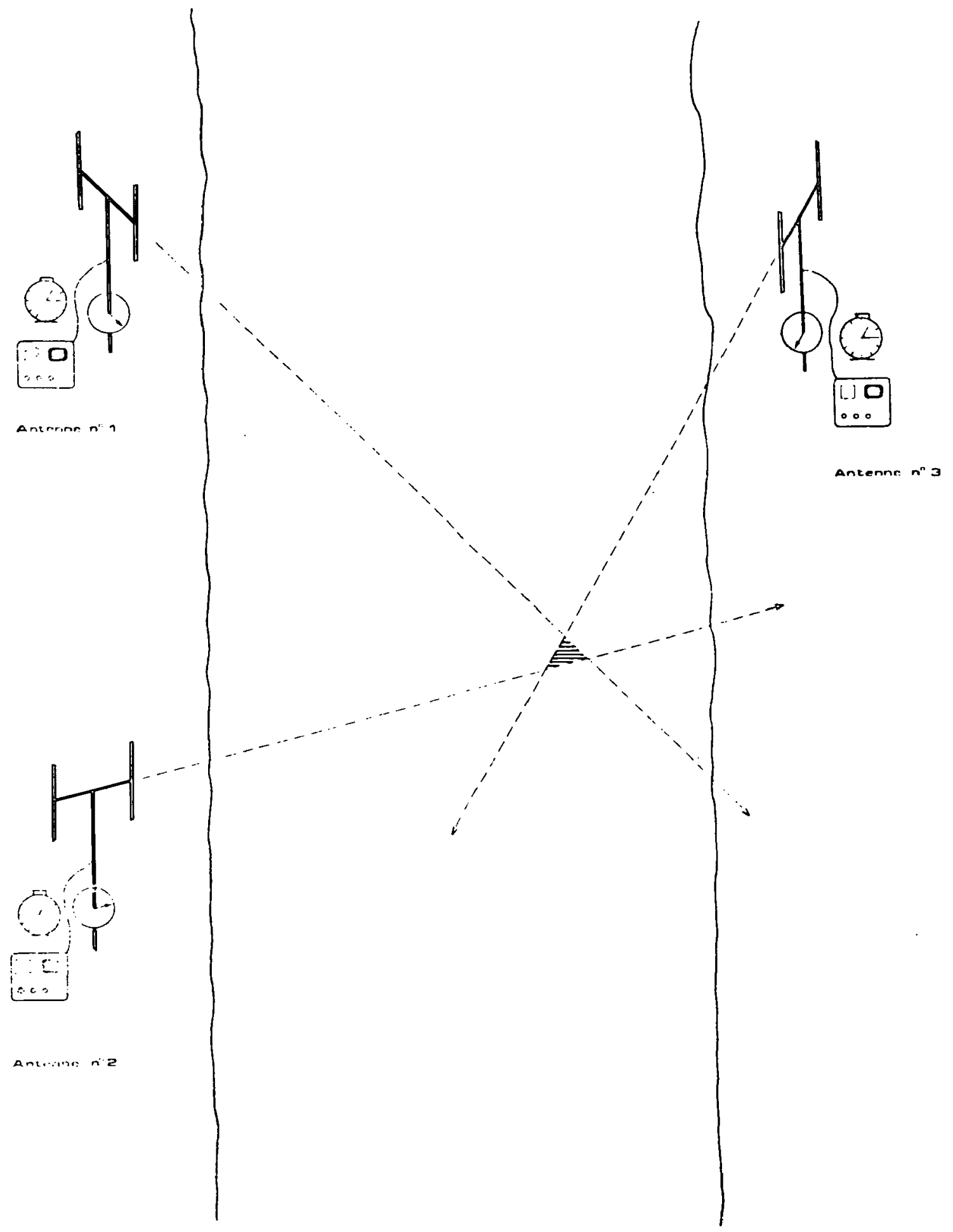

Figure 1bis : Principe de la triangulation.

Figure 1 bis : Principle of the triangulation. 
Il est ainsi théoriquement possible de connaître le moment d'arrivée du saumon au pied de l'obstacle, ses éventuelles prospections ainsi que le moment et le lieu de franchissement.

2.2. Description des deux sites d'études - Dampierre-en-Burly et Belleville-sur-Loire Les deux sites étudiés sont distants de $37 \mathrm{~km}$ (Figure 2 et 3 ).
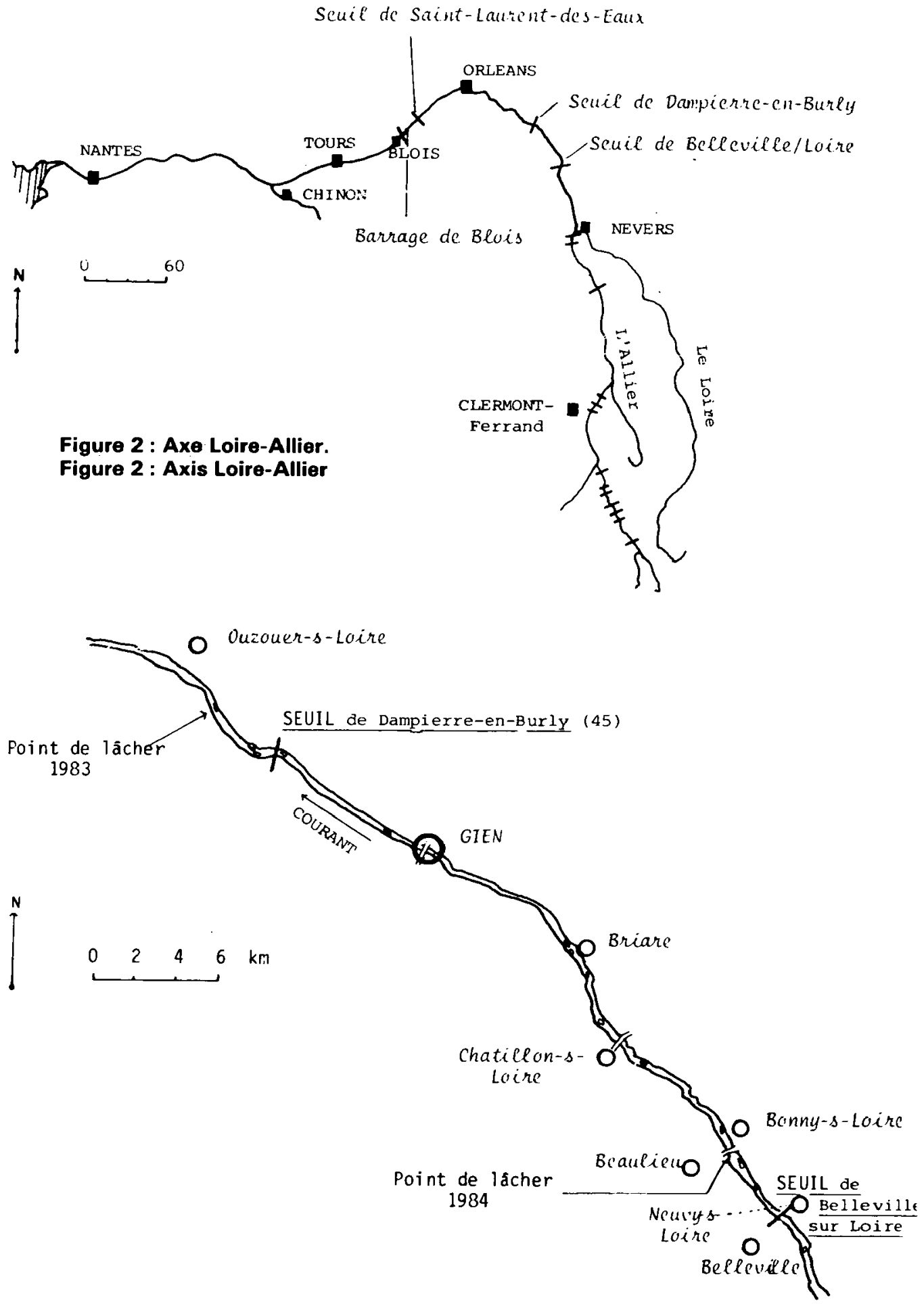

Figure 3 : Tronçon de Loire où les saumons ont été suivis.

Figure 3 : Section of the River Loire where the salmons have been radio-tracked. 
- Le seuil de Dampierre-en-Burly (Figure 4) est un barrage situé à $430 \mathrm{~km}$ de l'estuaire. II a une hauteur moyenne de 1,20 m à l'étiage pour une largeur de 6 mètres et une longueur de $210 \mathrm{~m}$. II est constamment recouvert d'une lame d'eau déversante s'écoulant à une vitesse de plusieurs mètres par seconde.

Une échancrure de 1,5 m de longueur a été ménagée en rive droite en guise de passe rustique pour faciliter le franchissement par les migrateurs dans certaines conditions de débit.

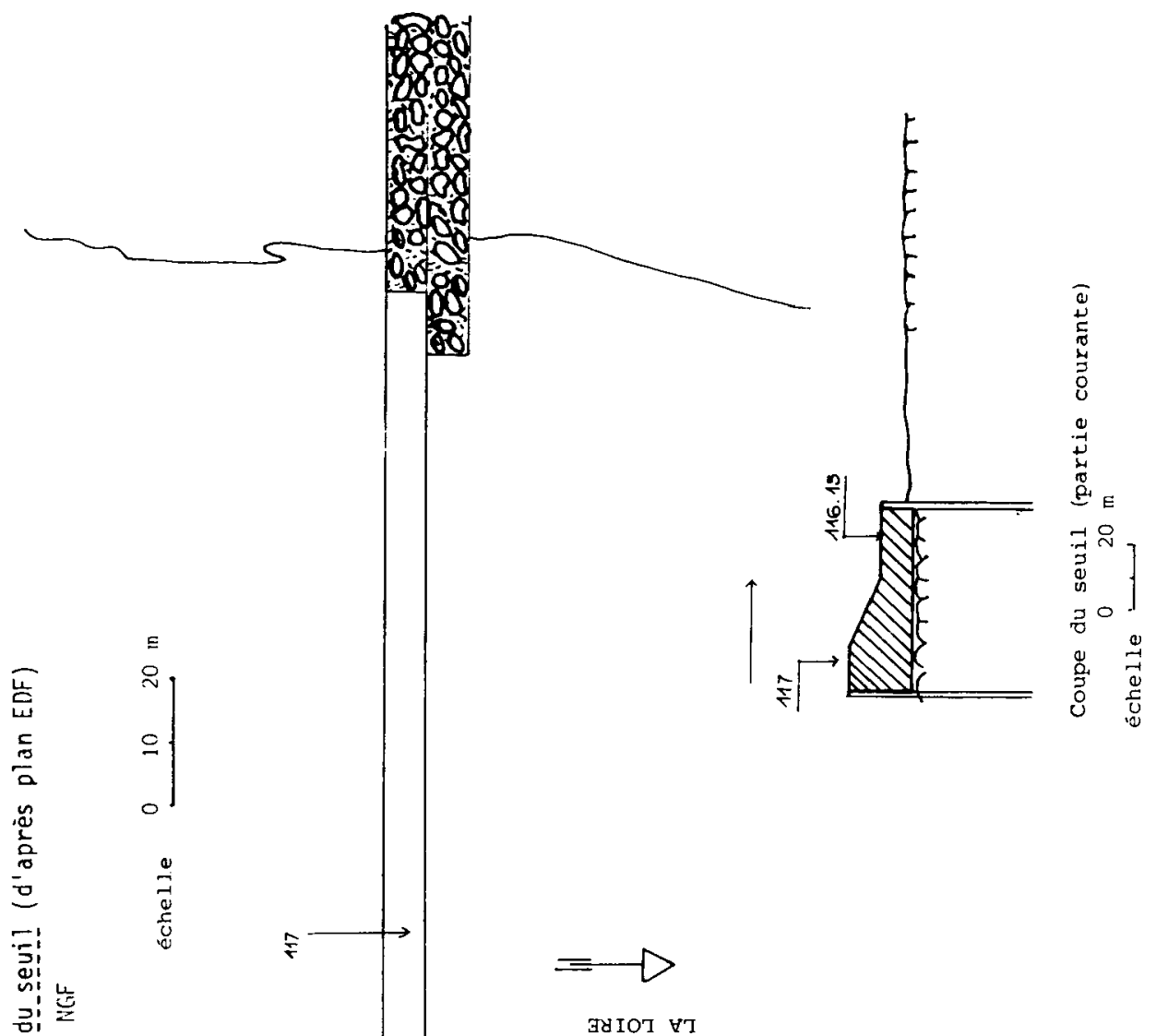


- Le seuil de Belleville-sur-Loire (Figure 5), de $370 \mathrm{~m}$ de longueur et 1,5 m de hauteur moyenne a l'étiage, est un barrage du même type que le précédent. Il en diffère par la présence d'une portion de seuil à section rectangulaire en rive gauche ainsi que par l'existence de deux passes à poissons à fentes verticales, dimensionnées, plus spécialement, pour les aloses (Alosa alosa).

\subsection{Marquage des saumons}

Tous les saumons, à l'exception d'un saumon piégé dans une des passes à poissons du seuil de Belleville-sur-Loire, ont été capturés par un pêcheur professionnel au moyen d'un filet barrage (filet non maillant tendu perpendiculairement à la rive et destiné à guider les saumons vers un carrelet mancuvré à partir d'un bateau). Le filet barrage a été installé à 4 kilomètres à l'aval du seuil de Dampierre-en-Burly du 15 janvier au 15 mars 1983, puis à hauteur de Saint-Dyé (amont de Blois) pour la suite de la campagne.

- Au total, dix-sept saumons ont été capturés et équipés d'un émetteur-radio (7 en 1983 et 10 en 1984). Deux présentaient un état sanitaire apparent médiocre. La taille moyenne de ces saumons était de $87 \mathrm{~cm}$ pour un écart type de $6,5 \mathrm{~cm}$.

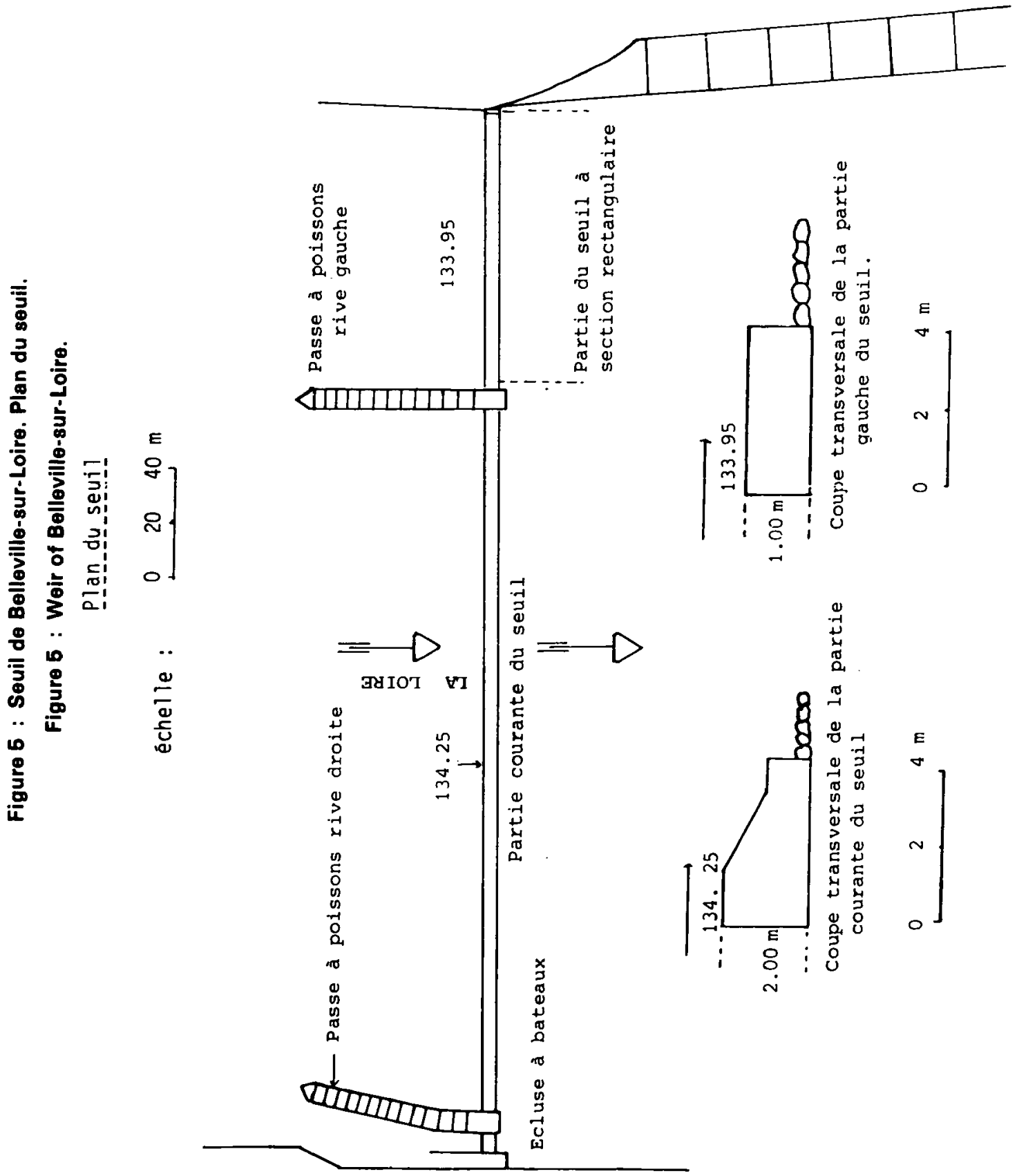


La fixation de l'émetteur-radio est faite par insertion stomacale selon la technique utilisée par le Fish and Wild Life Service en Alaska (Carl BURGER, 1982, communication personnelle).

Dès sa capture, le saumon est transféré dans un bac contenant de l'eau de Loire et un anesthésiant (MS 222). Quelques minutes plus tard, le saumon anesthésié est maintenu à la surface de l'eau, ventre en l'air. L'émetteur-radio (marque SMITH ROOT - $\phi=16 \mathrm{~mm}$, longueur : $55 \mathrm{~mm}$ ou $75 \mathrm{~mm}$ ) est alors poussé dans l'estomac du poisson au moyen de deux tubes en plastique introduits dans l'œsophage (cf. Figure 6). L'émetteur a été préalablement lubrifié avec de la glycérine.
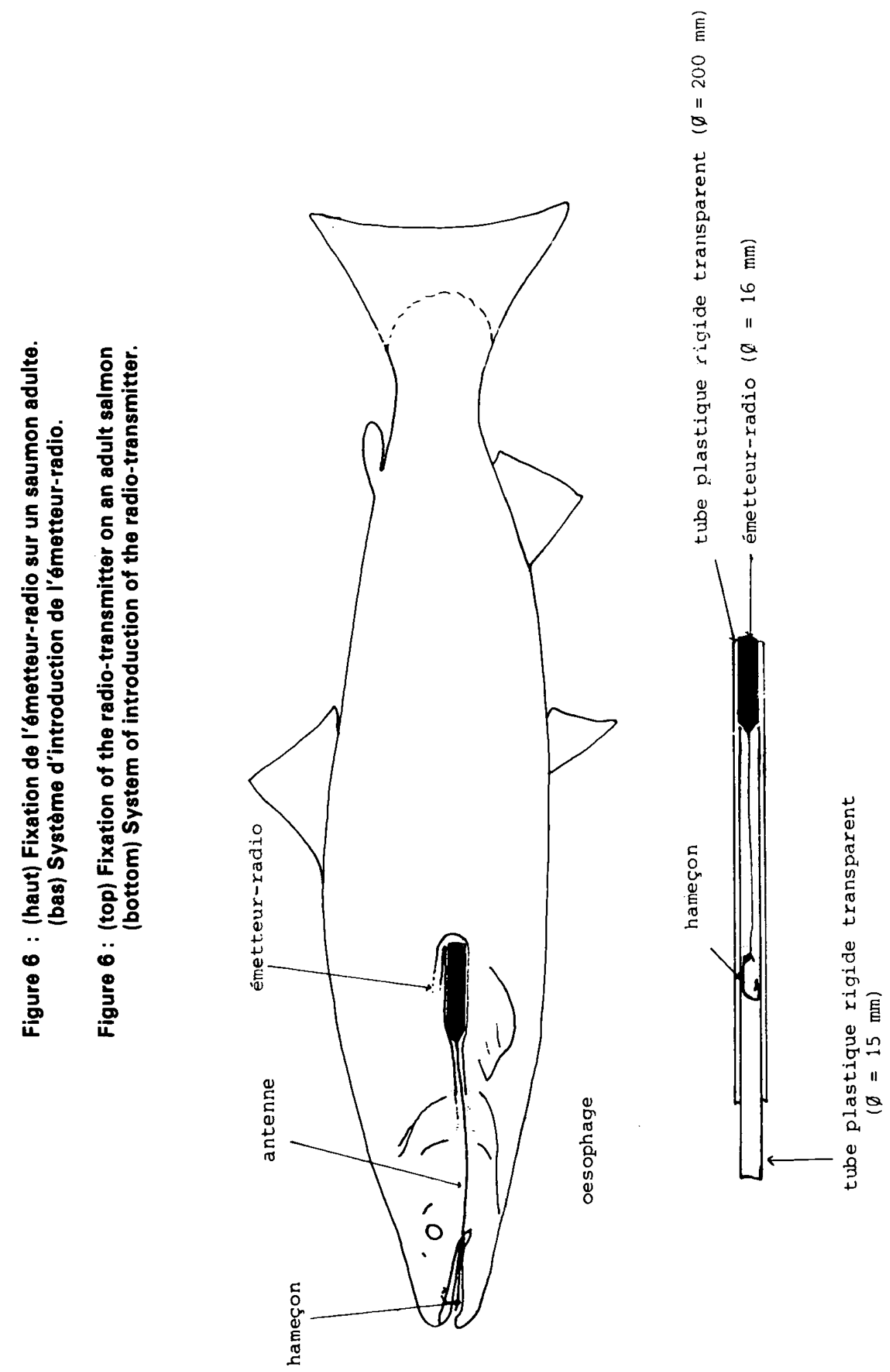
Photo 1 : Station mobile de repérage (récepteur monocanal et antenne-boucle).

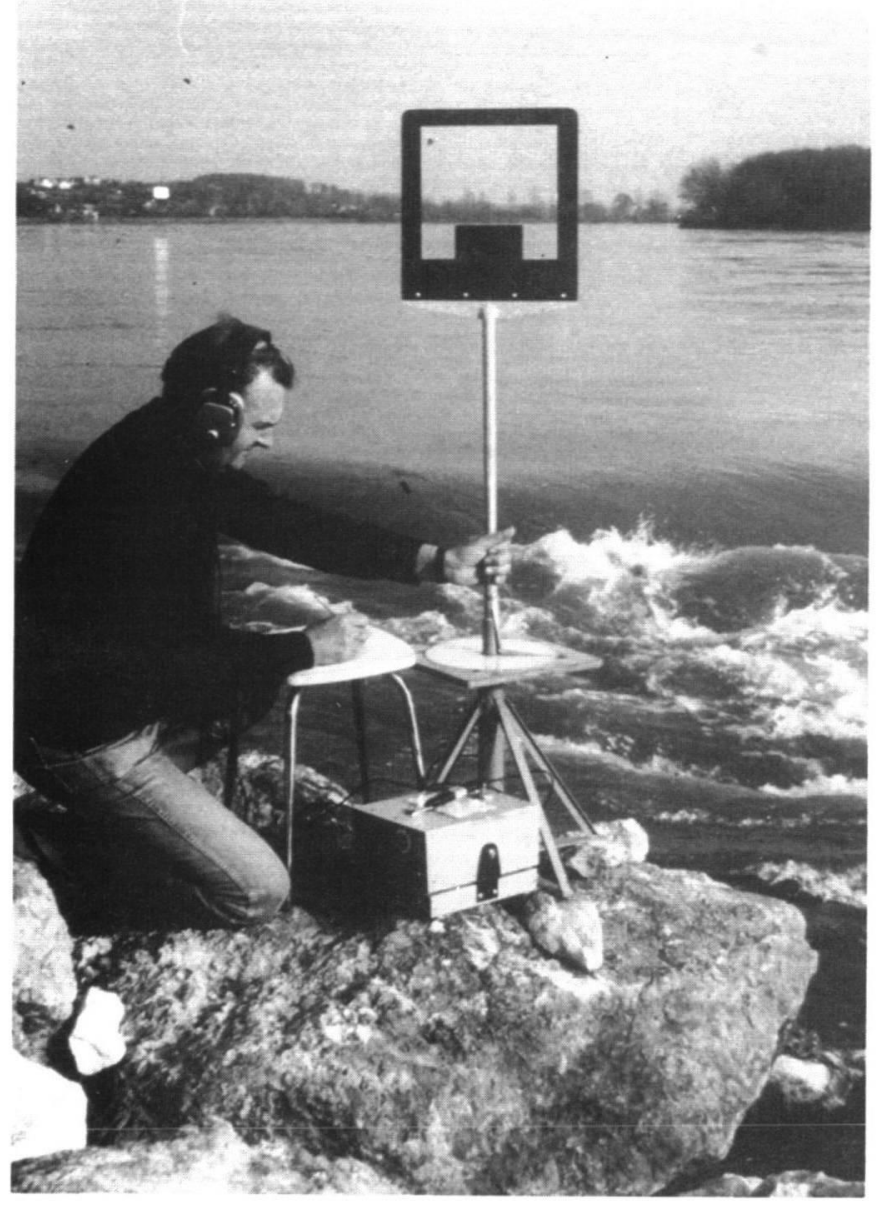

\section{Photo 1 : Mobile station of localization (mono channel receiver} and loop antenna).
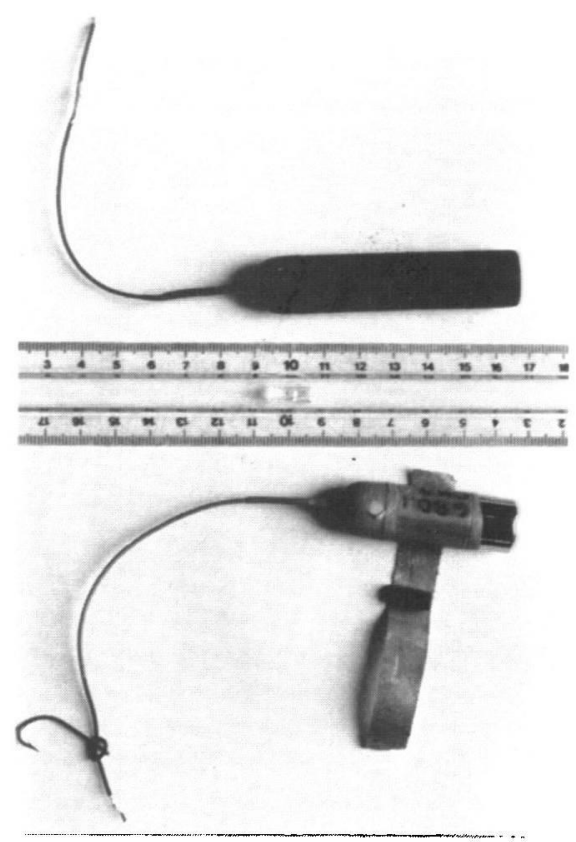

Photo 3 : Emetteurs-radio.

Photo 3 : Radio-transmitters.

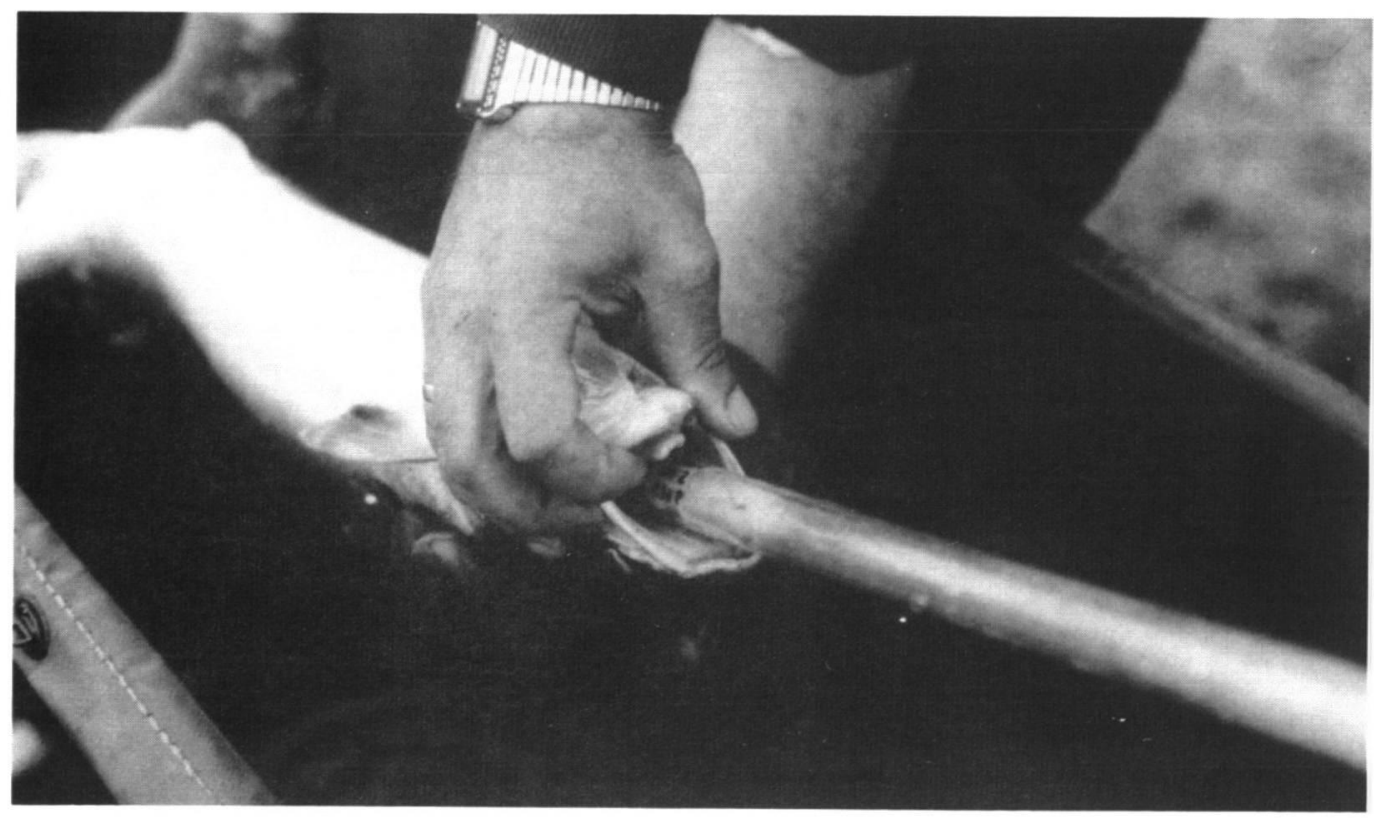

Photo 2 : Insertion stomacale de l“émetteur (saumon n 7 ).

Photo 2 : Stomachal introduction of the radio-transmitter (salmon $n^{\circ} 7$ ). 
L'extrémité de l'antenne est ensuite fixée au palais du saumon au moyen d'un hameçon (hamecon $n^{\circ} 6$ ).

Une étiquette Floy-tag est également insérée dans la musculature dorsale du poisson pour permettre son identification en cas de recapture.

Le temps nécessaire pour l'opération de marquage est inférieur à la minute.

Le saumon est ensuite transféré dans un bac de stabulation contenant de l'eau de Loire jusqu'à ce qu'il retrouve une certaine activité (environ $\mathbf{1 5}$ minutes plus tard), avant d'être relâché.

- Lors de la campagne d'étude 1983, les cinq premiers saumons ont été libérés quelques dizaines de minutes après le marquage, à proximité du lieu de capture, dans une zone calme ("bouge") située à $4 \mathrm{~km}$ à l'aval du seuil de Dampierre-en-Burly.

Les saumons capturés à Saint-Dyé, $120 \mathrm{~km}$ aval de Dampierre-en-Burly, ont été marqués sur le bateau puis transportés par voie terrestre jusqu'au point de lâcher situé à 4 km à l'aval du seuil de Dampierre-en-Burly en 1983 et à environ 5 km à l'aval du seuil de Belleville-sur-Loire en 1984.

\subsection{Suivi des saumons}

Les récepteurs-radios utilisés sont des récepteurs monocanal (SMITH-ROOT) fonctionnant dans la bande 40-41 $\mathrm{MHz}$.

- A Dampierre-en-Burly en 1983, deux stations de réception fixes (antenne de type "yagi" ou antenne de type "adcok") ont été installées en rive, une au droit du seuil pour déterminer le moment du franchissement et une autre, $120 \mathrm{~m}$ à l'aval, pour déterminer la zone de franchissement. Un poste de réception mobile (antenne de type "boucle") a été utilisé en complément pour les pointages par triangulation.

- Au seuil de Belleville-sur-Loire, en 1983, seuls deux postes de réception mobiles ont été utilisés pour suivre les saumons. En 1984, le matériel utilisé, identique à celui installé à Dampierre-enBurly, a été complété par un récepteur à 10 canaux (SMITH-ROOT) préréglé sur les fréquences des émetteurs-radios utilisés. Ce récepteur, connecté à un enregistreur, a été placé sur l'une des passes à poissons du seuil de Belleville-sur-Loire de telle sorte qu'il puisse indiquer le moment d'un franchissement lorsque les repérages radio étaient suspendus.

La progression entre les seuils a été suivie à partir de repérages périodiques au moyen de postes de réception mobiles (antenne boucle montée soit sur un bateau, soit sur un véhicule diésel tout terrain, soit sur un avion).

La fréquence de ces repérages a été fonction de la disponibilité du personnel puisque devaient être assurés simultanément la capture et le marquage des saumons, le suivi des saumons au pied des seuils ainsi que la progression des autres saumons marqués.

Durant ces deux campagnes de radio-pistage, l'évolution de la température de l'eau a été suivie à partir de mesures ponctuelles ou à partir des relevés des thermographes E.D.F. de Dampierre-enBurly et de Belleville-sur-Loire.

Les valeurs de débits provenaient de la station de jaugeage de Gien (Limniphone) située à environ $10 \mathrm{~km}$ à l'amont du seuil de Dampierre-en-Burly, (débit moyen interannuel proche de $\left.330 \mathrm{~m}^{3} / \mathrm{s}\right)$.

\subsection{Contrôle des pièges des deux passes à poissons du seuil de Belleville-sur-Loire.}

Les deux pièges a ménagés à l'extrémité amont des deux passes à poissons ont été périodiquement contrôlés (en moyenne 4 fois par jour et 6 jours par semaine) du mois de janvier au mois de maî 1984.

\section{RESULTATS}

Les résultats obtenus sont synthétisés dans les tableaux I et II et figures 7, 8 et 9.

A Dampierre-en-Burly, sur les sept saumons équipés d'émetteurs-radios en 1983, un seul a dévalé après sa libération. Ce saumon (S 7), qui présentait un état général médiocre, a régurgité son émetteur-radio dans les $\mathbf{4 8}$ heures qui ont suivi le lâcher.

En 1983 et 1984, à Belleville-sur-Loire, le comportement de dix saumons a pu être observé. Les sept autres saumons marqués n'ont pu être suivis soit en raison de la régurgitation de l'émetteurradio, soit en raison de la perte du signal-radio probablement liée à une défection de l'émetteur-radio. 
Tableau 1 : Tableau récapitulatif des comportements des saumons radio-pistéset desparamètres hydrologiques au seuil de Dampierre-en-Burly (1983).

Table 1 : Summary table of radio-tracked salmon's behaviour and of hydrological parameters at the weir of Dampierre-en-Burly (1983).

\begin{tabular}{|c|c|c|c|c|c|c|c|c|}
\hline$\stackrel{\circ}{ }$ & $\begin{array}{l}\infty \\
\stackrel{0}{0} \\
\vdots \\
\vdots\end{array}$ & 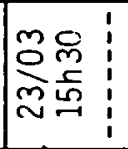 & 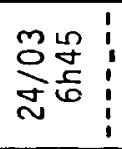 & 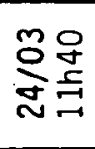 & $\underset{\frac{f}{d}}{\frac{n}{d}}$ & $\stackrel{\sim ్ N}{N}$ & 요 & $\begin{array}{ll}n & 0 \\
0 & 0\end{array}$ \\
\hline$\stackrel{i n}{\sim}$ & 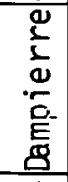 & 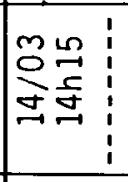 & 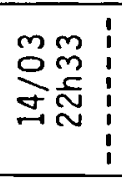 & 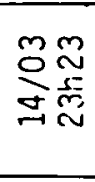 & $\begin{array}{l}\text { E } \\
E \\
\text { 요 }\end{array}$ & \& & 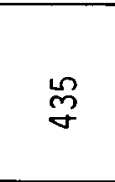 & $\begin{array}{ll}+ & + \\
0 & 0\end{array}$ \\
\hline 岕 & 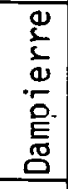 & 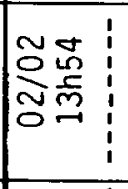 & 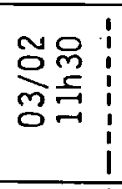 & 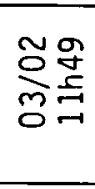 & $\begin{array}{l}\text { 들 } \\
\text { ᄋ }\end{array}$ & $\stackrel{尺}{N}$ & ్ㅗㅇ & $\begin{array}{ll}\sim & \sim\end{array}$ \\
\hline$\stackrel{m}{\sim}$ & 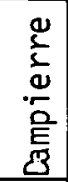 & : & 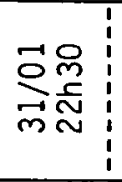 & 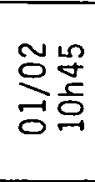 & 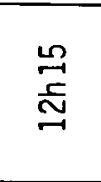 & $\stackrel{\leftarrow}{\sim}$ & $\stackrel{\text { ஜ }}{\forall}$ & $n$ \\
\hline$\tilde{N}$ & 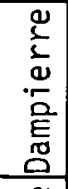 & 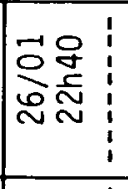 & 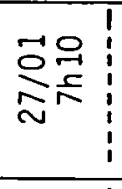 & $\begin{array}{l}\text { 묘요 } \\
\text { 옴 }\end{array}$ & $\begin{array}{l}\stackrel{ }{N} \\
\text { స్ల }\end{array}$ & 岕 & 总 & $\vec{n}$ \\
\hline$\vec{n}$ & 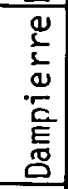 & 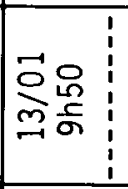 & 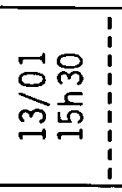 & 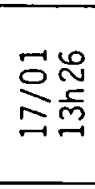 & $\begin{array}{l}\stackrel{n}{5} \\
.9 \\
+ \\
+\end{array}$ & \& & 苜 & $\begin{array}{l}n \\
n\end{array}$ \\
\hline $\begin{array}{l}-5 \\
2 \\
5 \\
n \\
5 \\
0 \\
5 \\
5 \\
0 \\
0\end{array}$ & 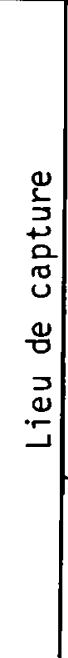 & $\underbrace{\underbrace{2}_{0}}$ & 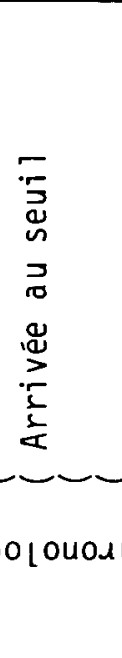 & 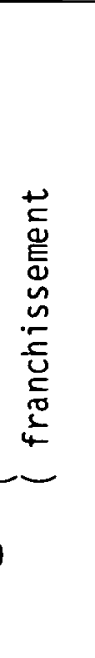 & 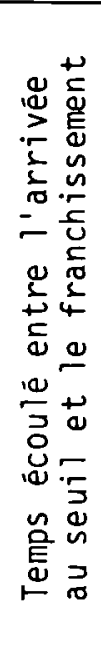 & 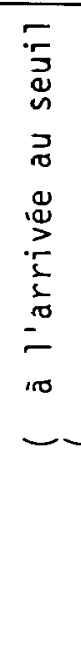 & 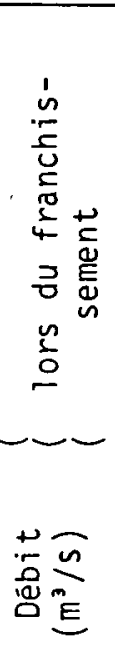 & 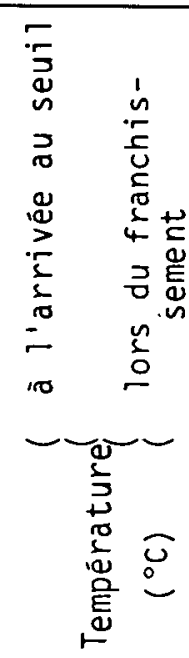 \\
\hline
\end{tabular}


Tableau II : Tableau récapitulatif des comportements des saumons radio-pistéset des paramètres hydrologiques au seuil de Belleville-sur-Loire (1983-1984).

Table II : Summary table of radio-tracked salmon's behaviour and of hydrological parameters at the weir of Belleville-sur-Loire (1983-1984).

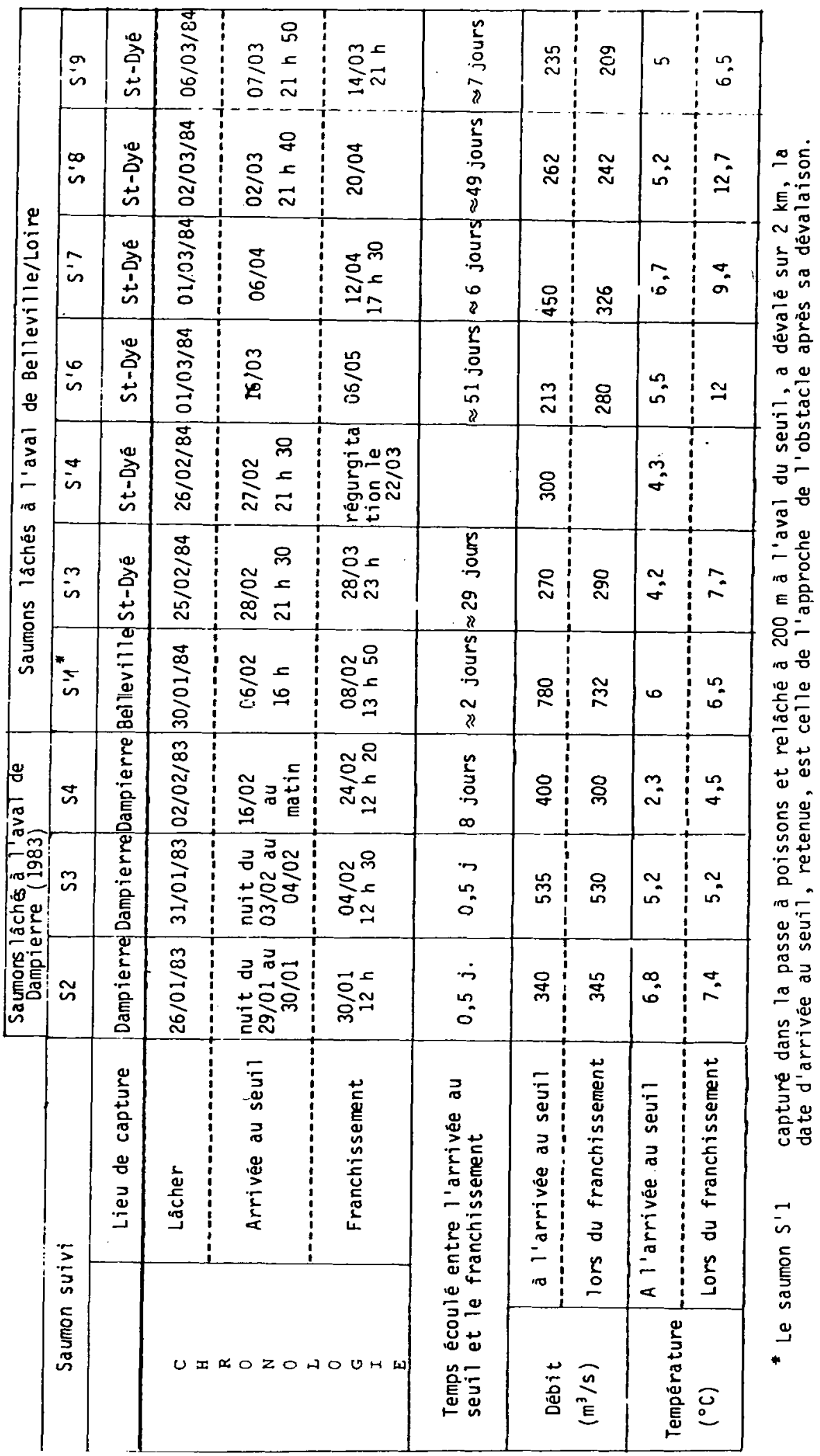


Figure 7 : Arrêt de migration au seuil de Dampierre-en-Burly et évolution des paramètres débit moyen et température moyenne (1983).

Figure 7 : Stop-over during migration at the weir of Dampierre-en-Burly and evolution of parameters average flow an average temperature (1983).

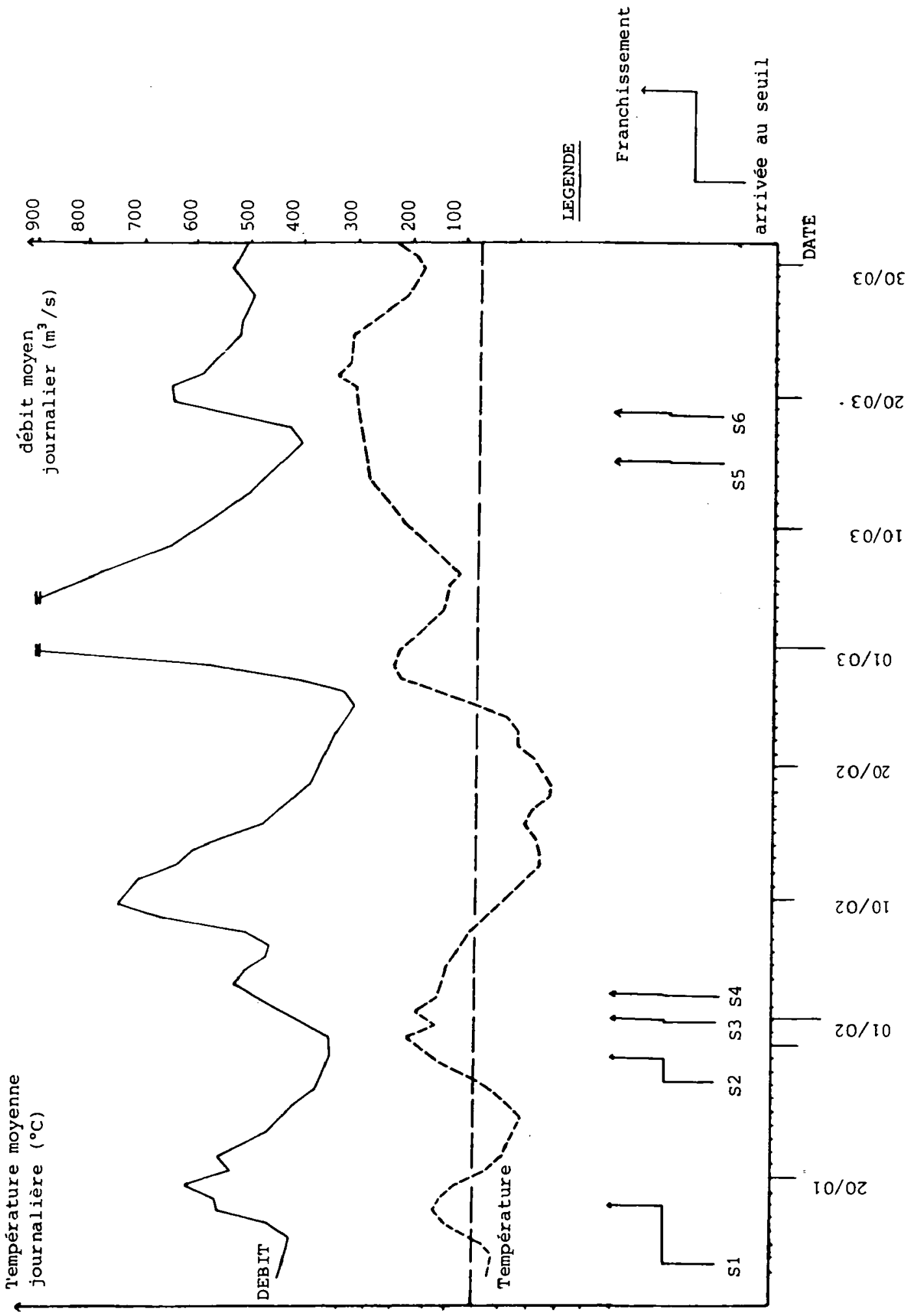

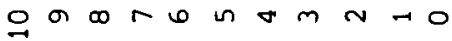


Figure 8 : Arrêt de migration au seuil de Belleville-sur-Loire et évolution des paramètres débit moyen et température moyenne (1984).

Figure 8 : Stop-over during migration at the weir of Belleville-sur-Loire and evolution of parameters average flow and average temperature (1984).

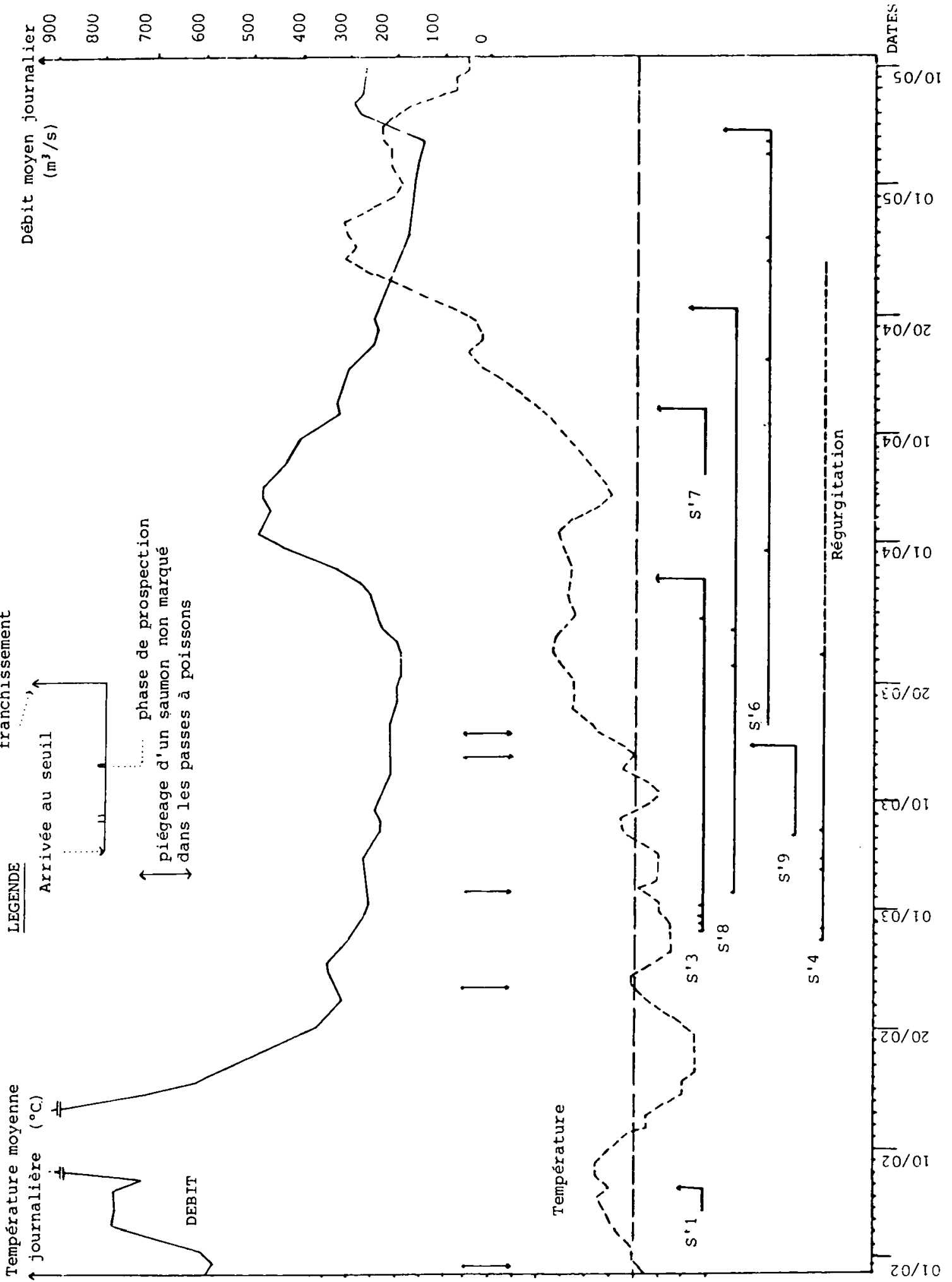

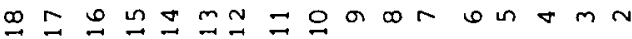


t.ameÉrature mojenne journalière $\left({ }^{\circ} \mathrm{C}\right)--$

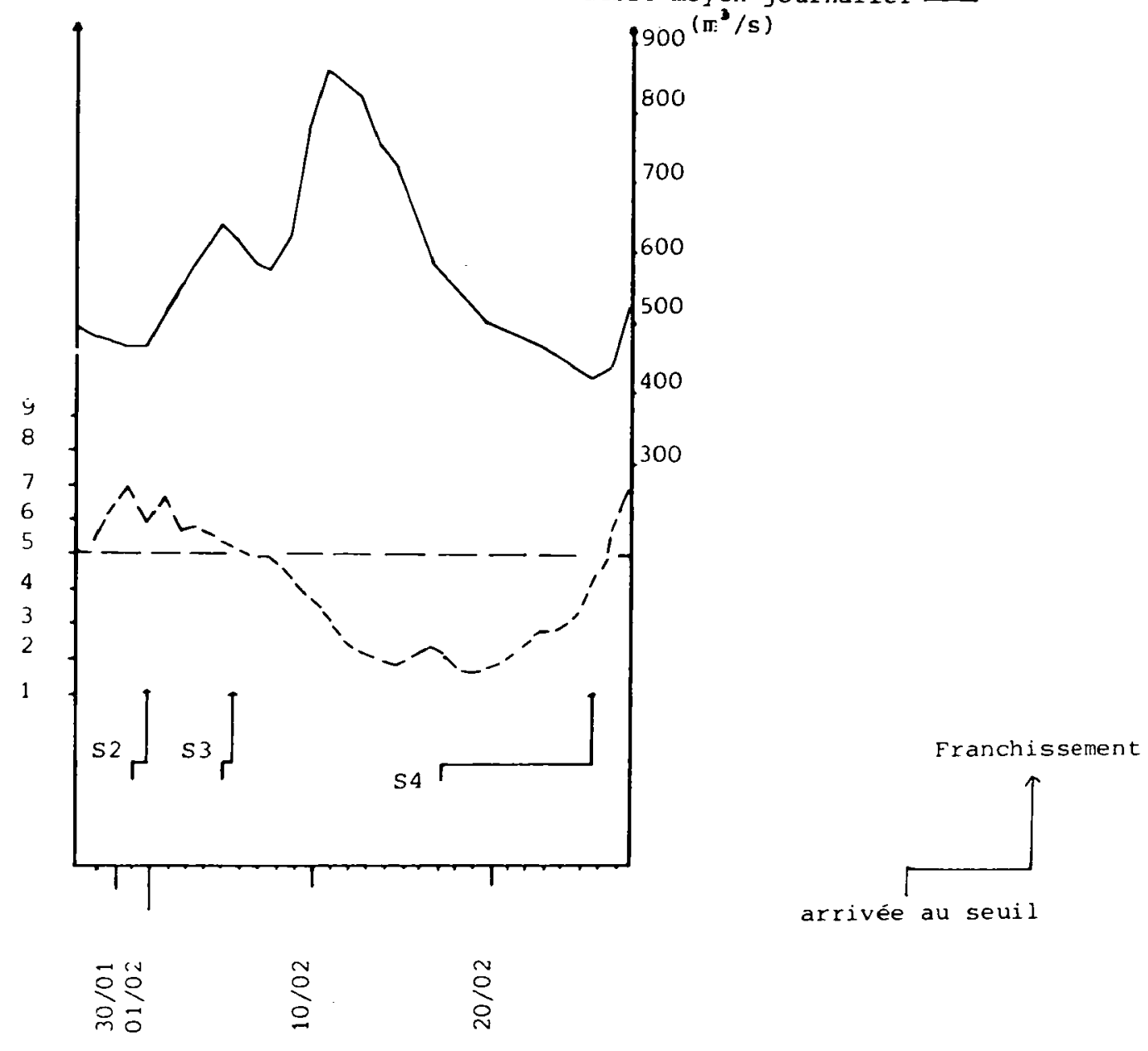

Figure 9 : Arrêt de migration au seuil de Belleville-sur-Loire et évolution des paramètres débit moyen et température moyenne (1983).

Figure 9 : Stop-over during migration at the weir of Belleville-sur-Loire and evolution of parameters average flow and average temperature (1983).

\section{DISCUSSION}

\subsection{Observations aux seuils}

Le comportement des six saumons suivis, à hauteur du seuil de Dampierre-en-Burly, a présenté certaines constantes.

- L'approche du seuil s'est faite le plus souvent par la rive droite de la Loire. Les saumons ont ensuite exploré l'obstacle, à l'exception du saumon $\$ 4$ qui a franchi directement le seuil.

- Les évolutions des saumons se faisaient principalement dans la moitié droite de la Loire, à une distance moyenne de 50 mètres du seuil (Figures 11 et 12, reconstitution des déplacements des saumons $\mathrm{S} 1$ et $\mathrm{S} 2$ pendant les phases de franchissement). Aucune dévalaison significative n'a été observée après l'arrivée devant le seuil. Les poissons se sont presque toujours maintenus dans les 150 premiers mètres à l'aval du seuil.

- Les deux saumons significativement retardés à hauteur du seuil (à partir de 24 heures) ont marqué des temps d'arrêt importants entre leurs prospections et notamment la nuit où seul un franchissement a été observé. Sur les cinq franchissements localisés, trois se sont produits en rive gauche. 
Figure 10 : Exemple de reconstitution des déplacements du saumon $\mathbf{S 1}$ pendant la phase de franchissement

Figure 10 : Example of simulation of the $\mathbf{S 1}$ salmon's routes during the phase of ascent of the weir.

- point clé du trajet

- point d'immobilisation

$\longrightarrow$, cheminement simplifié confirmé par les points intermédiaires

_- - cheminement extrapolé (pointages intermédiaires manquants).

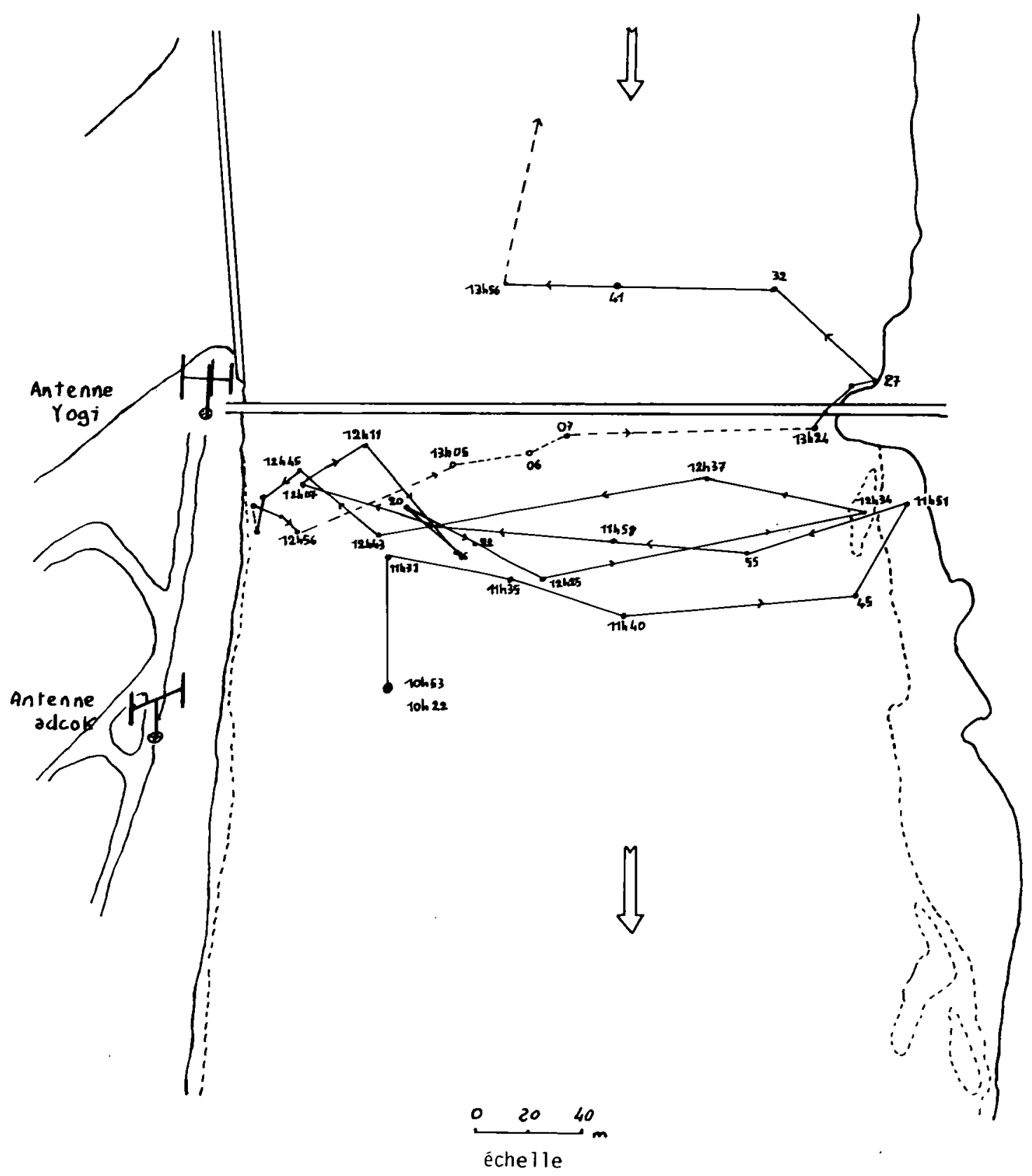


Figure 11 : Exemple de reconstitution des déplacements du saumon $\mathbf{S 3}$ pendant la phase de franchissement

Figure 11 : Example of simulation of the $\mathbf{S 3}$ salmon's routes during the phase of escent of the weir.

- point clé du trajet

- point d'immobilisation

$\longrightarrow$ cheminement simplifié confirmé par les points intermédiaires

- - cheminement extrapolé (pointages intermédiaires manquants).

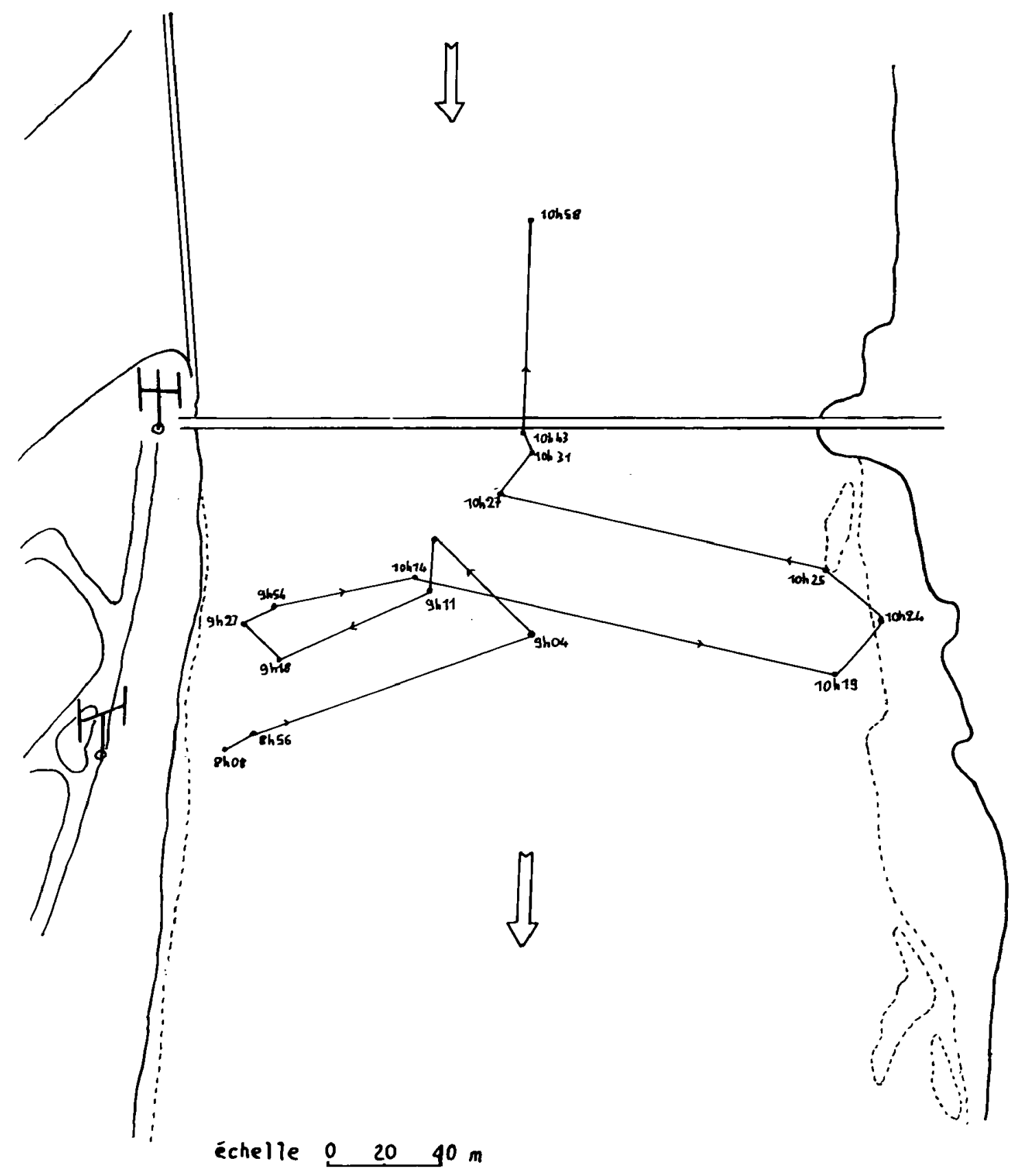


- Dans les conditions hydrauliques rencontrées (350 à $555 \mathrm{~m}^{3} / \mathrm{s}$ ), le facteur débit n'apparaît pas avoir été déterminant dans les retards observés. L'examen des données thermiques montre que les deux retards significatifs constatés (S1 et S2) correspondent à des températures basses lors de l'arrivée au seuil, $\left(=5^{\circ} \mathrm{C}\right)$. Le franchissement a eu lieu après que la température de l'eau se soit élevée au dessus de $6,5^{\circ} \mathrm{C}$. Les retards observés pour des températures d'arrivée au seuil, supérieures à $6^{\circ} \mathrm{C}$, sont comparativement faibles.

Les comportements des saumons suivis à hauteur du seuil de Belleville-sur-Loire présentent également des analogies.

- Dès l'arrivée au seuil, les saumons ont explorél'obstacle puis ont gagné des zones de repos situées plusieurs centaines de mètres à l'aval du seuil ( $800 \mathrm{~m}$ en moyenne), ces phases d'attente étant entrecoupées de nouvelles prospections au pied du seuil.

- Quatre franchissements ont pu être localisés dont trois en rive gauche. Les retards observés ont été très variables, de 0,5 à 8 jours en 1983.contre 2 à 51 jours en 1984.

L'étude des graphiques, représentant l'arrêt de migration au pied du seuil et l'évolution des paramètres débit et température, montre:

- que des franchissements se sont produits alors qu'au même moment d'autres saumons marqués. déjà bloqués depuis plusieurs jours par le seuil, demeuraient en phase d'attente.

- que le franchissement du seuil est souvent lié à une élévation de la température moyenne journalière durant les jours précédents (excepté $\left.S^{\prime} 6\right)$.

- qu'un seul saumon (S'6) a franchi le seuil dans des conditions de température et de débit qu'il avait déjà rencontrées durant l'attente à l'aval de l'obstacle.

- aucun franchissement n'a été observé pour des débits inférieurs à $220 \mathrm{~m}^{3} / \mathrm{s}$.

\subsection{Fiabilité de la méthode}

Aucun phénomène de dévalaison ou d'attente prolongée n'a été observé pour les cinq saumons marqués et libérés sur le lieu de capture. Par contre, plusieurs saumons, parmi ceux capturés à Saint-Dyé et transportés par camion jusqu'au point de lâcher, ont présenté des comportements laissant suspecter un effet de stress.

- Dévalaison sur plusieurs kilomètres pour 3 saumons (S7, S'6, S'7).

- Arrêt de migration de plusieurs jours sans raison apparente, immédiatement après le franchissement du seuil de Dampierre-en-Burly pour le saumon S6.

- Séjour prolongé à l'aval du seuil de Belleville-sur-Loire, malgré des conditions apparemment favorables au franchissement pour les saumons $S^{\prime} 4, S^{\prime} 6, S^{\prime} 7, S^{\prime} 8$.

En fait, la technique du radio-pistage peut engendrer trois type de stress ; les traumatismes liés à la capture, aux manipulations (marquage et transport) et au port de l'émetteur (SOLOMON, TJSTORETON-WEST, 1983).

- Dans le protocole de marquage utilisé, le stress lié à la capture a propablement été minime pour les saumons qui ont été anesthésiés quelques secondes après leur capture au carrelet, de même que le stress lié à la fixation stomacale de l'émetteur-radio, et ce, en raison du faible rapport poids de l'émetteur-radio sur poids du poisson (SOLOMON, TJSTORETON-WEST, 1983).

- En fait, les phénomènes apparents de stress n'ont été constatés que sur les saumons transportés en cuve pendant plusieurs heures, avant leur libération 110 ou $150 \mathrm{~km}$ plus en amont. Aussi, les manipulations liées au transport, qui s'accompagnent de modifications brutales des caractéristiques de l'eau de la Loire lors de sa libération susceptibles de désorienter le saumon, semblent être à l'origine de ces phénomènes de stress.

L'hypothèse d'un stress consécutif aux manipulations et notamment au transport est proposée pour expliquer le comportement des saumons S' 6 et S'8. Lors de leur séjour à l'aval du seuil, ils ont rencontré des conditions apparemment favorables (température en hausse $>7^{\circ} \mathrm{C}$ et débit en hausse $\Longrightarrow 500 \mathrm{~m}^{3} / \mathrm{s}$ ) sans tenter de franchir l'obstacle, alors que pendant la même période d'autres saumons marqués franchissaient le seuil.

L'interprétation du comportement d'un saumon radio-pisté à hauteur d'un obstacle et notamment la détection d'un comportement biaisé peuvent être facilitées si ce saumon a pu être suivi sur une distance suffisante avant et après l'obstacle. L'impact réel d'un obstacle sur la migration d'un saumon radio-pisté peut alors être dans certains cas corrigé.

Par exemple, des arrêts de migration ont été observés, en conditions de basse température. sans qu'il y ait un obstacle (Figure 11). 
Figure 12 : Progression du Saumon no 4 entre le point de lâcher et le seuil de Belleville-sur-Loire et évolution moyenne de la température et du débit.

Figure 12 : Advance of the $N^{\circ} 4$ salmon between the place of release and the weir of Bellevillesur-Loire and evolution of the average temperature and the average flow.

\author{
(Seuil A = Seuil de Dampierre-en-Burly) \\ (Seuil B = Seuil de Belleville-sur-Loire).
}
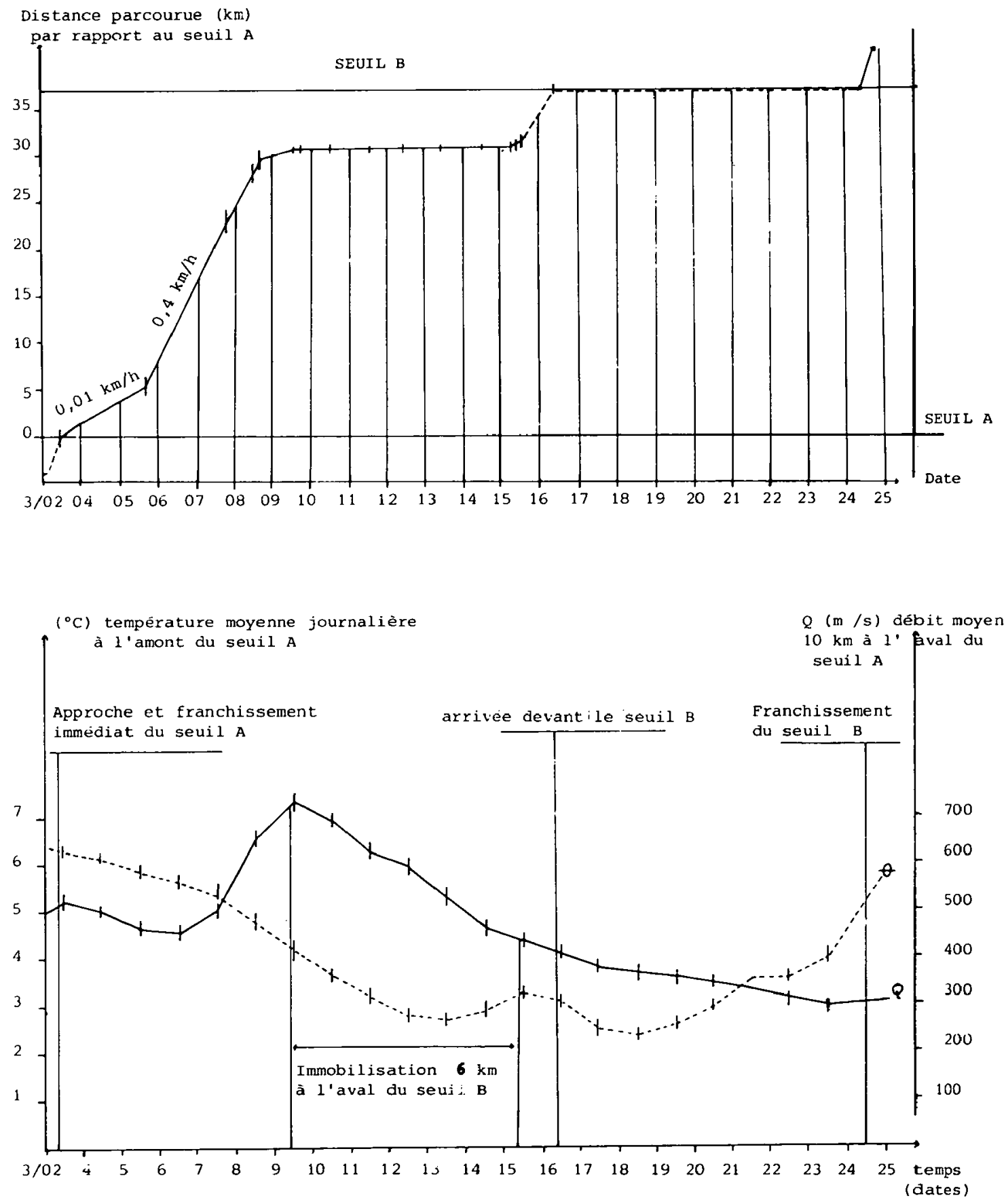
La vitesse moyenne de migration sur un tronçon de Loire sans obstacle, calculée à partir de mesures ponctuelles sur 11 saumons, pour des températures moyennes journalières supérieures à $3,5^{\circ} \mathrm{C}$, a été proche de $0,78 \mathrm{~km} /$ heure soit environ $19 \mathrm{~km} /$ jour (Tableau III). Les vitesses les plus

\begin{tabular}{|c|c|c|c|c|c|c|c|c|c|c|c|c|c|c|}
\hline $\mathrm{N}^{\circ} \mathrm{du}$ Saumon & $s^{\prime} 3$ & S1 & $S^{\prime} 4$ & S4 & S3 & S1 & $S^{\prime} 1$ & S4 & S3 & S2 & $S^{\prime} 1$ & S6 & S5 & $S^{\prime} 8$ \\
\hline $\begin{array}{l}\text { Vitesse moyenne } \\
\text { en } \mathrm{km} / \mathrm{h}\end{array}$ & 0,7 & 0,6 & 0,8 & 0,1 & $p, 3$ & 0,4 & 1,2 & 0,4 & 0,6 & 1,1 & 0,6 & 0,4 & 1,7 & 1,6 \\
\hline $\begin{array}{l}\text { Température moyen } \\
\text { ne journalière } \\
\text { de } l_{\text {'eau }}^{\circ} \mathrm{C}\end{array}$ & 3,5 & 4,5 & 4,5 & 4,6 & 5 & 5,5 & 5,5 & 5,5 & 6,5 & 7 & 7,5 & 8 & 10 & 14 \\
\hline $\begin{array}{l}\text { Distance parcou- } \\
\text { rue en } \mathrm{km}\end{array}$ & 4,7 & 3,5 & 4,7 & 5 & 9 & 37 & 4,7 & 23 & 37 & 37 & 3,1 & 24 & 7 & 75 \\
\hline
\end{tabular}

Tableau III : Vitesses moyennes horaires de progression par rapport à la rive, enregistrées pour différents saumons radio-pistés (arrêts éventuels compris).

Table III : Average speed (kilometer per hour) of advance recorded for different radio-tracked salmons (eventual stops included).

élevées observées correspondent à des températures $=10^{\circ} \mathrm{C}$. Le saumon $\mathrm{S}^{\prime} 8$, dont le comportement a été considéré comme biaisé à l'aval de l'obstacle, a progressé après,le franchissement du seuil de Belleville-sur -Loire à une vitesse moyenne de $37 \mathrm{~km} /$ jour sur une distance de $75 \mathrm{~km}$. Ce saumon a été recapturé par un pêcheur à la ligne, son émetteur-radio était toujours en place. Le stress était donc probablement effacé au moment du franchissement.

Le suivi simultané de plusieurs saumons sur une distance suffisante devrait permettre une détection plus fiable des comportements biaisés.

\subsection{Importance des facteurs température et débit}

En Loire moyenne, le facteur température et le facteur débit ont globalement une évolution inverse en début et en fin de période de migration. A l'automne, les débits tendent à augmenter et la température baisse, alors qu'au printemps, les débits décroissent et la température augmente.

Ces deux facteurs peuvent donc devenir limitants à tour de rôle. Leur évolution conjointe peut aboutir à des valeurs propices au franchissement lorsque la vitesse maximale de nage du saumon qui est fonction de la température (WARDLE, 1975 in BEACH, 1984) est compatible avec les difficultés physiques du franchissement qui varient avec le débit.

Plusieurs auteurs ont déjà signalé l'existence d'un seuil thermique en dessous duquel le saumon atlantique ne tente pas de franchir l'obstacle. Ce seuil thermique a été évalué à $5,5^{\circ} \mathrm{C}$ sur la rivière Tays par MENZIS (1939) (in BANKS, 1969) et confirmé dans les passes à poissons du barrage de Pitlochry (Ecosse), PYEFINLK (1965), (in BANKS, 1969). THIOULOUSE (1972) indique qu'il n'a observé aucun franchissement d'obstacle entre 0 et $4^{\circ} \mathrm{C}$, que les tentatives échouaient entre 5 et $6^{\circ} \mathrm{C}$ et qu'à partir de $7^{\circ} \mathrm{C}$ les saumons franchissaient l'obstacle. HELLAWELL, LEATHAM, WILLIAMS (1974) l'ont estimé, compris entre 5 et $5,5^{\circ} \mathrm{C}$ sur la rivière Frome en Ecosse. CUINAT (1980) $1^{\circ}$ estime proche de $7^{\circ} \mathrm{C}$ sur l'Allier.

Par ailleurs, à Belleville-sur-Loire (1984), nous avons constaté que les seules captures de saumons effectuées dans les pièges des passes à poissons l'ont été, alors que la température de l'eau était comprise entre 5 et $6^{\circ} \mathrm{C}$ et le débit entre 220 et $580 \mathrm{~m}^{3} / \mathrm{s}$. Aucun de ces saumons (au nombre de six) n'était marqué.

Les observations faites sur les saumons radio-pistés, en Loire moyenne en 1983 et 1984 , confirment donc l'existence d'un tel seuil thermique qui serait compris entre 5 et $6^{\circ} \mathrm{C}$.

L'impact des seuils de Dampierre-en-Burly et de Belleville-sur-Loire sur la migration des saumons se traduit par un retard de migration dont la durée (en condition de débit moyen) serait liée à celle de la période où la température est inférieure au "seuil thermique". Des faibles débits, en conditions thermiques favorables, peuvent également engendrer des retards. Ces conditions que l'on rencontre peu en hiver deviennent par contre plus fréquentes en fin de période de migration. 
Le cumul des retards que peuvent occasionner ces deux seuils réduit en fait les chances qu'ont les saumons de rencontrer des débits favorables pour franchir les autres obstacles situés plus en amont dans l'Allier. L'impact de ces deux seuils affecterait notamment les saumons qui pénètrent au début du printemps dans l'estuaire.

\section{CONCLUSion}

La technique du radio-pistage, avec insertion stomacale de l'émetteur-radio, s'est révélée fiable pour observer le comportement des saumons adultes (Salmo salar) a condition que la libération du saumon, après marquage, soit faite dans une zone calme à proximité du lieu de capture.

Cette technique a permis de reconstituer les déplacements de plusieurs saumons à l'aval d'un barrage et de déterminer la durée de l'arrêt de migration ainsi que le moment et le lieu de franchissement. Des franchissements ont pu être constatés de nuit.

Dans les conditions de débit rencontrées, la franchissabilité des seuils de Dampierre-en-Burly et de Belleville-sur-Loire par des saumons équipés d'émetteurs-radio a surtout été fonction de l'évolution du facteur température. essentielles :

Cette technique développée à une plus grande échelle peut apporter des informations

- pour apprécier les modalités de franchissement d'un obstacle

- pour connaître les conditions d'attractivité des passes à poissons.

- pour définir la longueur des réserves à l'aval des barrages, suivant les zones préférentielles de "stationnement" qui sont spécifiques à chaque site.

Un nombre suffisamment important des saumons $(<30)$ doit être équipé d'un émetteur-radio pour accroître le nombre d'observations simultanées dans des conditions données et compenser les éventuelles régurgitations.

\section{BIBLIOGRAPHIE}

BANKS J.W., 1969. A review of the literature on the upstream migration of adult salmonids. J. Fish Biol, 1, 85136.

BEACH M.H., 1984. Fish pass design - criteria for the design and approval of fish passes and other structures to facilitate the passage of migratory fish in rivers - Fisheries Research, technical report no 78, Lowestoft 46p.

CUINAT R., 1980. "Le saumon du Bassin Loire Allier" in Saumons, n 34 - 4ème semestre.

HELLAWELL J.M., LEATHAM H., WILLIAMS G.I., 1974. The upstream migratory behaviour of salmonids in the river Frome, Dorset, in J. Fish Biol 6, 729 - 744.

SOLOMON, TJSTORETON-WEST, 1983. Radio-tracking of migratory salmonids in rivers : development of an effective system. Fisheries Research, technical report $n^{\circ} 75$, Lowestoft $11 \mathrm{p}$.

THIOULOUSE G., 1972. Le comportement du saumon -Essai d'éthologie du saumon de l'Allier - Plein air service, Clermont-Ferrand - P. Eguillon imprimeur, 280 p. 